\title{
Performance analysis of an indoor localization and mapping system using 2D laser range finder sensor
}

\author{
Mounia Janah \\ Department of Electrical and Computer Engineering \\ Yokohama National University \\ Yokohama, Japan \\ mounjanah@gmail.com
}

\author{
Yasutaka Fujimoto \\ Department of Electrical and Computer Engineering \\ Yokohama National University \\ Yokohama, Japan \\ fujimoto@ynu.ac.jp
}

\begin{abstract}
There is several implementations of techniques for solving the SLAM problem that is a process to expand autonomous mobile robots field. In this context, we will be comparing swarm algorithms for solving the simultaneous localization and mapping problem such as Firefly Algorithm, Particle Swarm Optimization and Glowworm Swarm Optimization, we directed a group of experiments using a wireless system composed of 2D laser range finder UTM-30LX, iRobot Roomba 600 and Raspberry Pi 3 as an experimental environment.

Keywords-SLAM, PSO, FA, GSO, Raspberry Pi 3
\end{abstract}

\section{INTRODUCTION}

The general simultaneous localization and mapping (SLAM) approach is now a well recognized and approved part of robotics, asks a mobile robot to establish a map and localize it's position simultaneously [5].

SLAM problem can be resolved in different forms, we can use a huge combination of various hardware [2] [3]. There are a couple steps in SLAM can be implemented using some varied algorithms since it's considered like a concept [4] [7]. Simultaneous localization and mapping still has high potential for research and a lot of works proposed a different localization and mapping approachs and presented a different ways of achieving the extreme goal of obtaining a global map [13]. This paper propose to analyse different swarm algorithms used in SLAM process, a test problems and experiments was used to compare between them and will be presented applying these algorithms to solve SLAM problem.

\section{SWARM ALGORITHMS USED IN SLAM PROCESS}

\section{A. Particle Swarm Optimization (PSO)}

In the particle swarm optimization algorithm, the particle can change its velocity and its position in the search range. The algorithm establish a range of possible solutions which particles are flown through a problem space

(1) is the velocity of the $i^{t h}$ particle at the $k^{t h}$ step and (2) it's position :

$$
v_{i}^{k}=w v_{i}^{k-1}+c_{1} r_{1}\left(p_{i}-x_{i}^{k-1}\right)+c_{2} r_{2}\left(p_{n}-x_{i}^{k-1}\right)
$$

$$
x_{i}^{k}=x_{i}^{k-1}+v_{i}^{k}
$$

For the $i^{t h}$ particle at the $k^{t h}$ step of the particle swarm optimization algorithm, $v_{i}^{k}$ and $x_{i}^{k}$ define velocity and position respectively, $p_{i}$ describe for the $i^{t h}$ particle the local best position and $p_{n}$ describe for all particles the global best location. $c_{1}$ and $c_{2}$ represent the acceleration coefficients parameters, and $r_{1}, r_{2}$ represent the arbitrary numbers drawn from an homogeneous distribution [8]. The inertia weight is the parameter $w$ was described to maintain how much the current velocity of the particle dedicate to it's velocity in the next iteration, it illustrate the balance adjusting between the global and local searchs [11].

The particle swarm optimization algorithm is demonstrated as below :

First step : At first iteration we put $k$ to 0 and we choose number of particles, weight coefficients of particle and maximum number of iterations respectively $m>2, w, c_{1}, c_{2}$, $K_{\max }$

Second step : We set velocity $v_{i}{ }^{0}$ for $\{i=1,2, \ldots, m\}$ to 0 and initialize a range of particles for $\{i=1,2, \ldots, m\}$. Where $i$ is the number of particles and $k$ is the iteration number. Initialize local best position to a state value of particles for $\{i=1,2, \ldots, m\}$. Set global best location to $\operatorname{argminf}\left(\right.$ pbest $\left._{i}\right)$ for $\{i=1,2, \ldots, m\}$ with pbest is the local best position.

Third step : By using evaluation function $f(x)$ we update local best position and global best location and we set pbest ${ }_{i}$ to $\operatorname{argminf}\left(x_{i}\right)$ for $\{i=1,2, \ldots, m\}$ and global best location to $\operatorname{argminf}\left(\right.$ pbest $\left._{i}\right)$ for $\{i=1,2, \ldots, m\}$.

Fourth step : We will finish the process if $k$ equal to $K_{\max }$ elseways we will return to the second step.

We decided that the following parameters are the right ones for the algorithm to converge better [12]:

- Particles number : $m=50$

- Weight coefficients: $w=0.729, c_{1}=1.4955, c_{2}=$ 1.4955

- Iterations number : $K_{\max }=600$ 


\section{B. Firefly Optimization Algorithm (FA)}

In the firefly algorithm, the deviation of attractiveness $\beta$ is given by [6]:

$$
\beta=\beta_{0} \mathrm{e}^{-\gamma r^{2}}
$$

Which $r$ is the distance and $\gamma$ is the fixed luminous absorption coefficient of the medium. The $i^{\text {th }}$ firefly move to a brighter $j^{\text {th }}$ firefly and that movement is decribed by :

$$
x_{i}^{t+1}=x_{i}^{t}+\beta_{0} \mathrm{e}^{-\gamma r_{i j}^{2}}\left(x_{j}^{t}-x_{i}^{t}\right)+\alpha_{t} \epsilon_{i}^{t}
$$

where $x_{i}^{t}$ and $x_{j}^{t}$ respectively at time $t$ represent the positions of firefly $i$ and firefly $j, \beta_{0}$ is the attractiveness at the distance $r$ equal to 0 , the randomisation parameter that can be tuned to rate the random component is described as $\alpha_{t}$ and the vector of random numbers is defined by $\epsilon_{i}^{t}$. This random vector is typically peaked from Gaussian or uniform distribution.

The firefly optimization algorithm is demonstrated as below:

First step : At first we set the weight coefficients $\alpha, \beta$ and $\gamma$, we define the objective function $f(x)$ where $x=$ $\left(x_{1}, x_{2}, \ldots, x_{d}\right)$ for $d$ dimension and the light intensity $I_{i}$ at $x_{i}$ is determined by $f\left(x_{i}\right)$

Second step : We give random locations for $N$ fireflies in $d$ dimension and initialize evaluation of all $N$ fireflies, After that, if the light intensity $I_{j}$ of firefly $j$ is strictly higher than the light intensity $I_{i}$ of firefly $i$, the firefly $i$ move towards firefly $j$ in $d$ dimension

Third step : We evaluate new solution and update light intensity and we check if the best position is found so far

Fourth step : We update the weight coefficient $\alpha$ and we try to sort the fireflies and if $k=K_{\max }$ we end the process and find the best firefly.

We decided that the following parameters are the right ones for the algorithm to converge better [12]:

- Fireflies number : $m=50$

- Weight coefficients: $\beta_{0}=0.2, \gamma=6.5, \alpha=\operatorname{rand}(0,1)$

- Iterations number: $K_{\max }=600$

\section{Glowworm Swarm Optimization (GSO)}

Glowworm swarm optimization (GSO) mimic the glowing aspect of glowworms, the advantages of this swarm algorithm are: the speed of convergence for finding the global optimized answer is very high and it can contribute with highly non linear problems [10]. The luciferin level defined in (5) is depending on the value of objective function and position of the glowworm.

$$
L_{i}(t)=(1-\rho) L_{i}(t-1)+\gamma F\left(p_{i}(t)\right)
$$

Which $L_{i}(t-1)$ represents the previous luciferin level for glowworm $i, \gamma$ is the luciferin enhancement fraction, $\rho$ is the luciferin decay and it's between the range of 0 and 1 $\in[0,1]$ and $F\left(p_{i}(t)\right)$ define the evaluation function at current glowworm $i$ position. Consequently, by using the condition (6) each glowworm $i$ examine its own neighbourhood to choose the neighbors that have the highest luciferin level

$$
N_{i}(t)=\left\{z: d_{i z}<r d_{i}(t) \operatorname{and} L_{z}(t)>L_{i}(t)\right\}
$$

Which $r d_{i}(t)$ represents the local decision range and $d_{i z}$ represents the Euclidean distance between glowworm $i$ and glowworm $z$ all neighbors probabilities are estimated using the following equation to determine the best neighbor from the neighbourhood range [14]:

$$
\operatorname{prob}_{i z}=\frac{L_{z}(t)-L_{i}(t)}{\sum_{k \in N_{i}(t)} L_{k}(t)-L_{i}(t)}
$$

each glowworm determined the movement direction and the glowworm which has the higher probability has a chance to be chosen from the neighborhood set. Using the equation (7) the position of the glowworm $p_{i}$ is adapted based on the position of the selected neighbor $p_{z}$ :

$$
p_{i}(t)=p_{i}(t-1)+s \frac{p_{z}(t)-p_{i}(t)}{d_{i z}}
$$

The local decision range $r d_{i}$ is adapted by the equation (9):

$$
r d_{i}(t)=\min \left\{r s, \max \left[0, r d_{i}(t-1)+\beta\left(n t-\left|N_{i}(t-1)\right|\right)\right]\right\}
$$

To restrain the neighborhood range size a constant parameter is used $n t$, a model constant $\beta$ and the radial sensor range constant $r_{s}$,

The glowworm swarm optimization algorithm is demonstrated as below [10] :

First step : Initialization of the parameters : we initialize a range of glowworm individual $p_{i}$, the population size $m$, the step size $s$, a number of iteration $K_{\max }$, the initial value of luciferin $I_{0}$, the initial value of the radial range $r_{0}$ and the weight coefficients

Second step : Solutions initializations : we set $k$ to 0 and randomly generate the initialize solutions $L_{i}(t)=I_{0}, r d_{i}=$ $r_{0}$, we calculate the value of objective function $F\left(p_{i}(t)\right)$

Third step : Iteration procedure: First of all, in the luciferin update phase we calculate $I_{i}(t)$ for each glowworm , in the movement phase we calculate $N_{i}(t)$ for each glowworm and $p_{i}(t)$ in the neighborhood for each glowworm $i$, we calculate $L_{i}(t+1)$ for each glowworm $i$

Fourth step : Decision range update : we calculate $r d_{i}$ for each glowworm , if $k=K_{\max }$ we end the process and return the best solution.

We decided that the following parameters are the right ones for the algorithm to converge better:

- Glowworm individuals number: $m=50$

- Weight coefficients: $\rho=0.4, \gamma=0.6, \beta=0.08$

- Maximum iterations number: $K_{\max }=600$ 


\section{SOLVING THE INDOOR LOCALIZATION AND MAPPING PROBLEM}

\section{A. SLAM process}

We change the basic data from the laser range finder sensor to an occupied grid-map, the value of the cell is described as $\operatorname{MAP}(x, y) \in\{0,1\}$ and it's initial value equal to 0 . And if there is an obstacle $\operatorname{MAP}(x, y)$ will be equal to 1 [11].

The position and head angle of the robot are respectively represented by $x_{0}, y_{0}$ and $\theta_{0} . \delta x, \delta y$ and $\delta \theta$ sampled data extracted from an LRF described the robots movement information [1].

We can evaluate the robots movement by matching the latest data to a map built from past data. The robot try to minimize Eq. (10) by using the optimization algorithms (PSO,FA or GSO).

$$
\begin{aligned}
f(z) & =\frac{N_{\text {valid }}-N_{\text {fit }}(z)}{N_{\text {valid }}} \\
N_{f i t} & =\sum_{i=1}^{n_{\text {max }}} M A P\left(x_{i}, y_{i}\right)
\end{aligned}
$$

where $\left(x_{i}, y_{i}\right)$ is the point coordinates on the map and $n_{\max }$ is the valid points number equal to 719 , with the robots movement is defined as $z=(\delta x, \delta y, \delta \theta)$ and the number of samples obtained from the sensor is described as $N_{\text {valid }}$ in each scan and range detection distance is from $30 \mathrm{~mm}$ to $30,000 \mathrm{~mm}$ [4].

\section{B. Simulation environment}

We will conduct our first test in the simulator tool Gazebo Fig.2 performing in ideal map and we set the following specifications of the sensor used in the simulator :

- detection distance : $30-30,000 \mathrm{~mm}$

- angle of the scan : $270 \mathrm{deg}$

- angular resolution : $0.25 \mathrm{deg}$
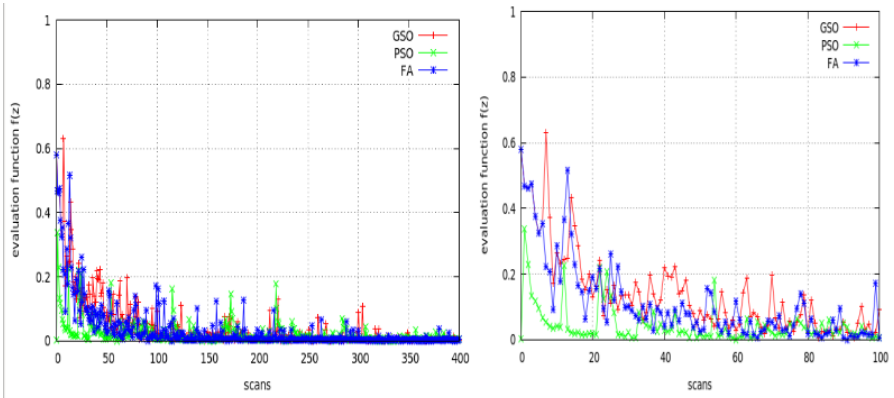

Fig. 1. Evaluation of the fitness function $f(z)$

We will test on the case when we do a translation and rotation and as the results shows in Fig. 3, Fig. 4 and Fig. 5 the PSO seems better in terms of the accuracy of the map , better than both swarm algorithms FA and GSO.
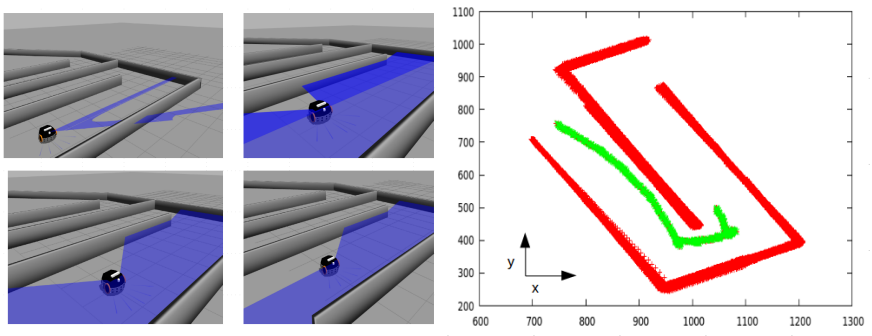

Fig. 2. Our environment in the Gazebo Fig. 3. Particle Swarm Optimization simulator result in Gazebo simulator
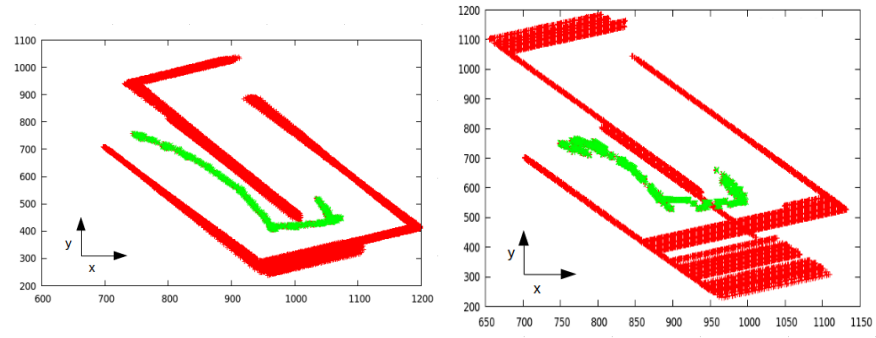

Fig. 4. Firefly Optimization Algorithm result in Gazebo simulator

Fig. 5. Glowworm Swarm Optimization result in Gazebo simulator

\section{System Overview}

Fig. 6 and Fig. 7 interpret the whole architecture of the integrated system. Raspberry $\mathrm{Pi} 3$ and Base Station that represent the personal laptop are the main parts of the system. We are using a wireless router for transfering data between these two parts. The Robot and sensor are connected to the Raspberry Pi 3 using USB cable and the Base Station is connected through WiFi.
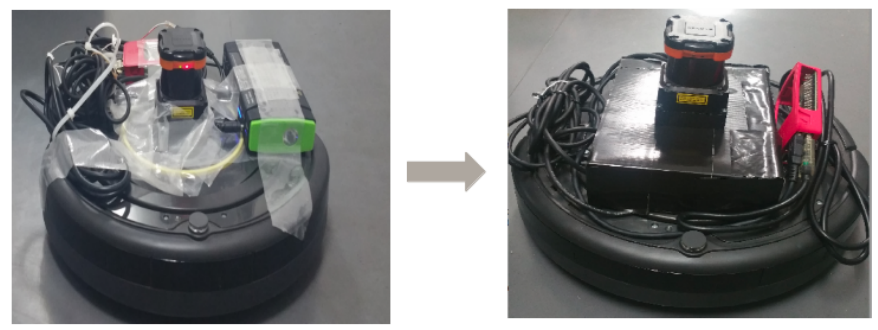

Fig. 6. Environment platform :2D laser range finder UTM- 30LX + iRobot Roomba 600 + Raspberry Pi 3

We are using 2D laser range finder UTM- 30LX, iRobot Roomba 600, Raspberry Pi 3 in the platform Fig. 8. We build the whole interface using SSH connection to connect with the personal laptop and using it to control everything directly from it . So at the end we have a bi-directional connectivity between all pairs of components on all ports and they should be in the same network.

To visualize the experimental environment in real time, we will be using rviz simulator contained in Robot Operating System (ROS) [9].

The system be publishing information about the exchange between coordinate frames, we run a tool for visualizing the 


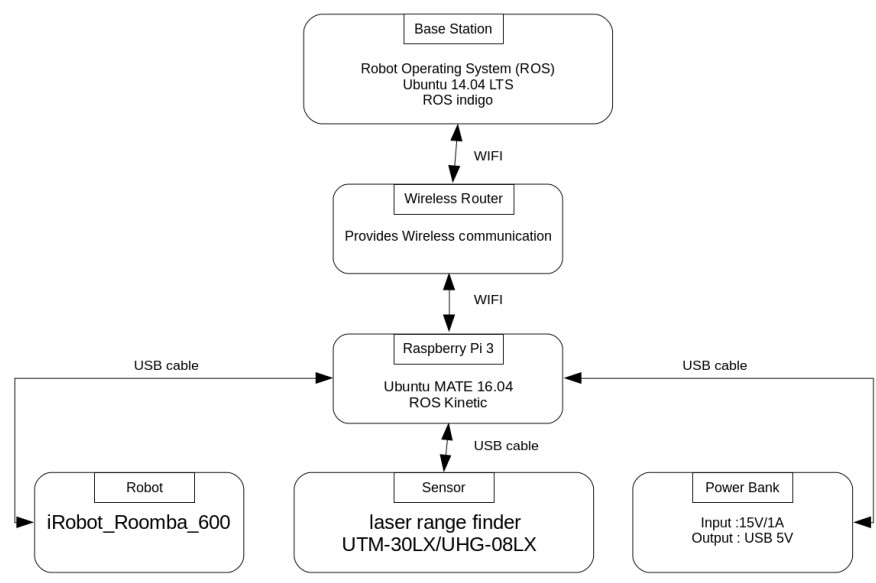

Fig. 7. Architecture of the integrated system
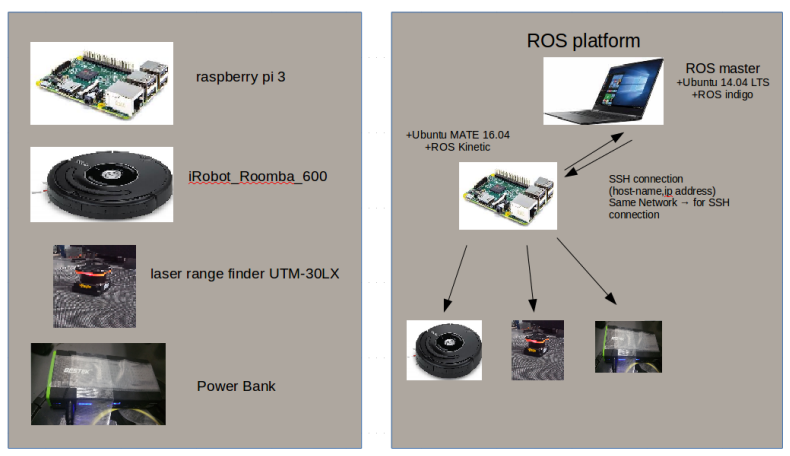

Fig. 8. Architecture of the environment platform

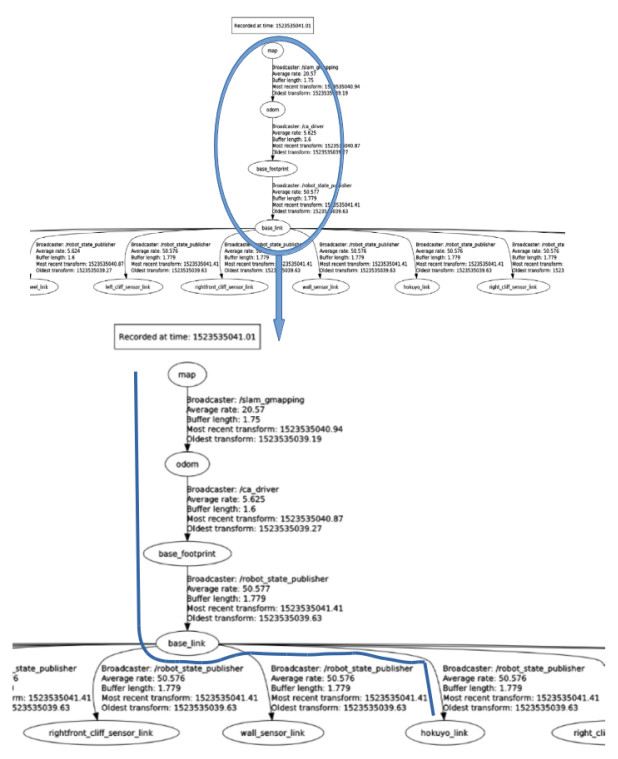

Fig. 9. Transform tree diagram

tree of frames Fig. 9 being broadcast over ROS platform, so we can afford the frames that are broadcasted, we can see that map frame is the parent of the rest of frames.

\section{Experimental Environments}
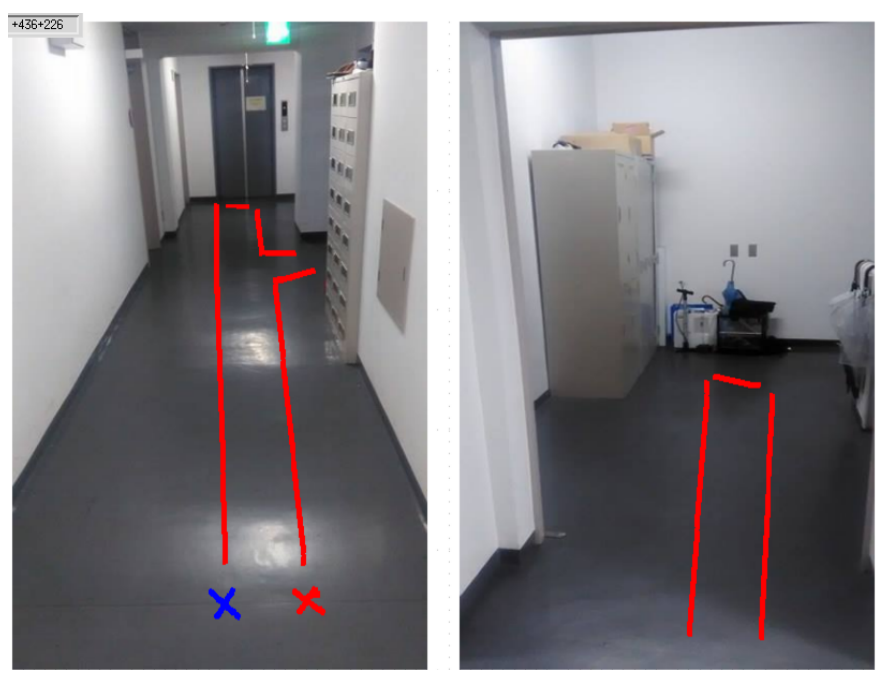

Fig. 10. Experimental Environment Example I
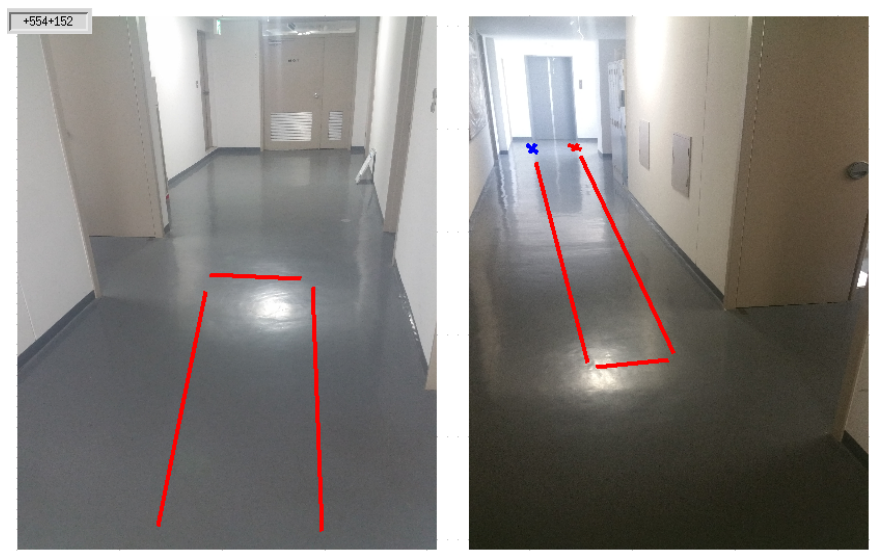

Fig. 11. Experimental Environment Example II

The experiments considered in this paper were conducted in two types of environments in the Department of Electrical and Computer Engineering building of the Yokohama National University Fig. 10, Fig. 11. The maps of these environments are shown in Fig. 12 and Fig. 13.

Laboratory Corridor 1 Fig. 10 : the length of the corridor has approximately $(10 \mathrm{~m})$, the floor is flat.

Laboratory Corridor 2 Fig. 11: the length of the corridor has approximately $(12 \mathrm{~m})$, the floor is flat.

\section{E. Results and analysis}

Procedure in the experimental environment example I Fig. 12: The robot moves forward by $7 \mathrm{~m}$ and rotates by $\frac{\pi}{2}$, after we moves forward by $4 \mathrm{~m}$ and rotates by $\pi$ comeback by 4 $\mathrm{m}$ and rotates by $\frac{\pi}{2}$ finaly the robot moves by $3 \mathrm{~m}$ and rotates by $\pi$ and moves forward by $10 \mathrm{~m}$ to reach final position .

Procedure in the experimental environment example II Fig. 13: The robot moves forward by $8 \mathrm{~m}$ and and rotates by $\pi$ comeback by $8 \mathrm{~m}$ to reach final position . 


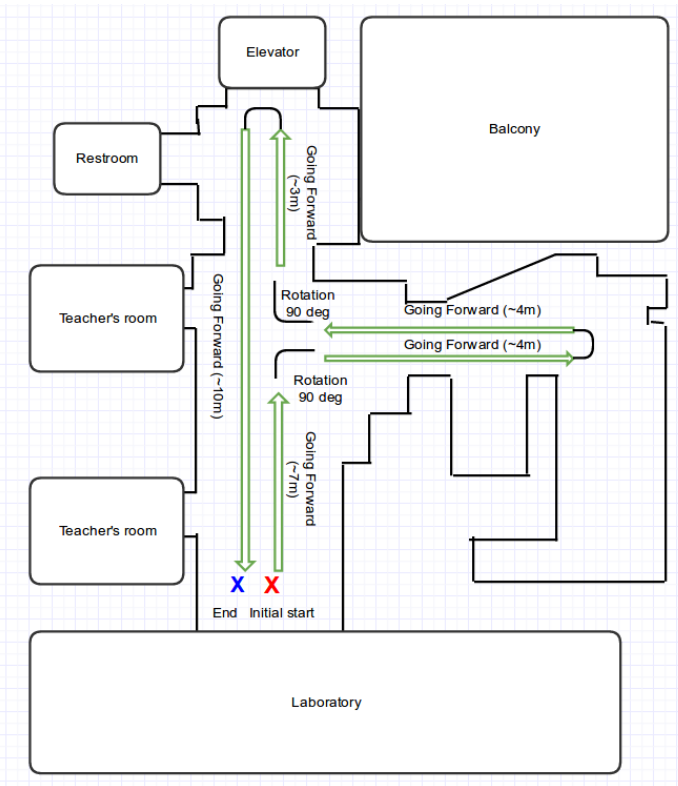

Fig. 12. The location map of Experimental Environment Example I

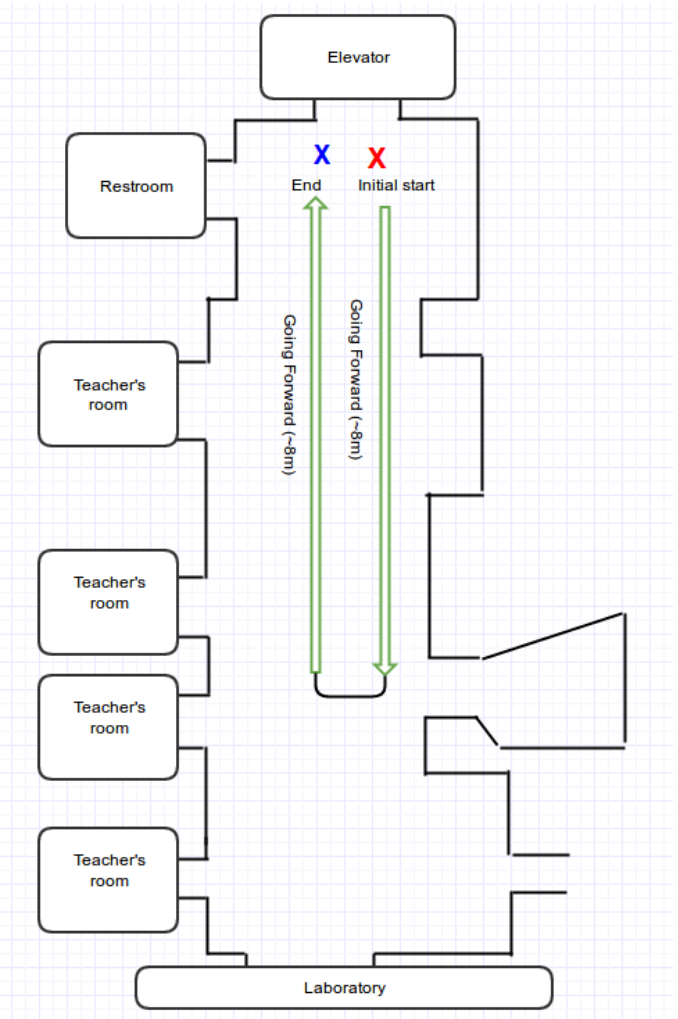

Fig. 13. The location map of Experimental Environment Example II

As the results shows in both experimental environment examples I and II Fig. 14, Fig. 15, Fig. 16, Fig. 20 Fig. 21 and Fig. 22 in terms of accuracy of the map and Fig. 17, Fig. 18, Fig. 19, Fig. 23, Fig. 24 and Fig. 25 in terms of the trajectory of the robot with same conditions PSO takes the lead better than both swarm optimization methods FA and GSO.
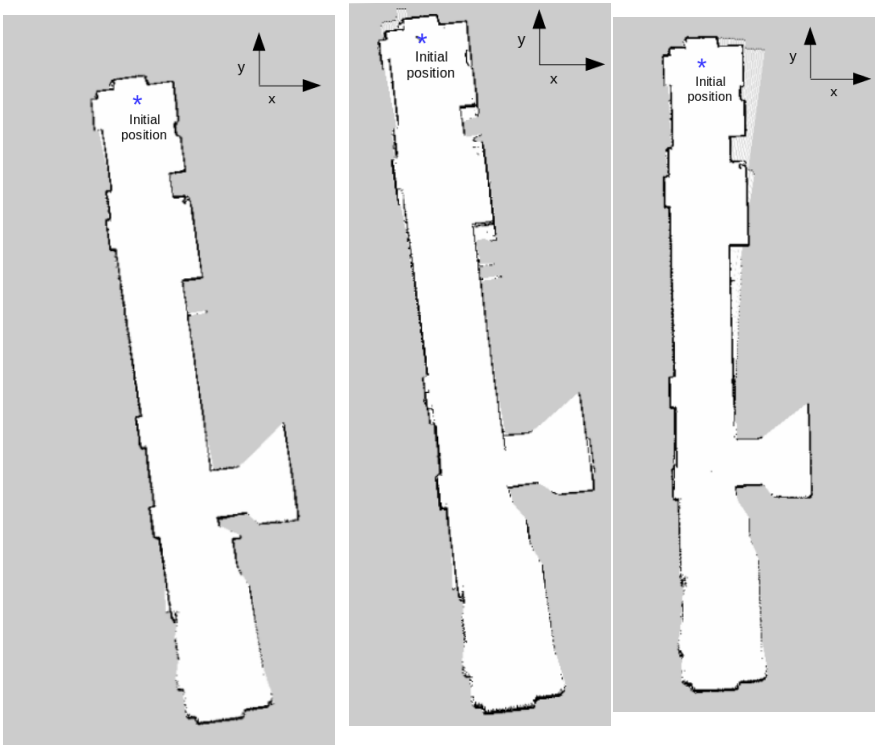

Fig. 14. Particle Swarm Optimization result in experimental environment II

Fig. 15. Firefly Opti- Fig. 16. Glowworm mization Algorithm re- Swarm Optimization sult in experimental en- result in experimental vironment II

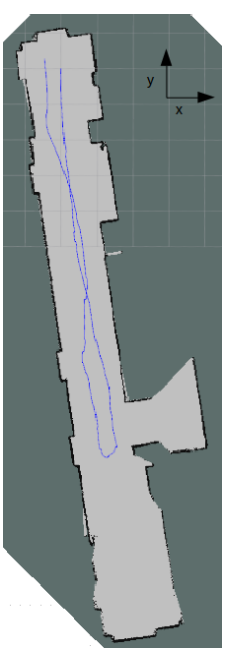

Fig. 17. Trajectory of the Particle Swarm Optimization result in experimental environment II

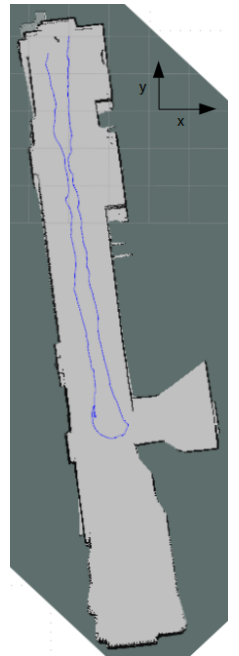

Fig. 18. Trajectory of the Firefly Optimization Algorithm result in experimental environment II

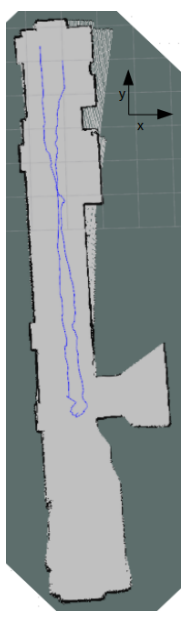

Fig. 19. Trajectory of the Glowworm Swarm Optimization result in experimental environment II
We evaluate the fitness function according to the number of iterations and number of scans Fig.1 and Fig. 26 and compare the evaluation between the swarm algorithms.

\section{CONCLUSIONS}

We compare between swarm procedures and inspected their equalities and differences. The position and orientation are estimated by using PSO, FA and GSO algorithms, simulation and experiment results prove that PSO performances exceeded both the optimization algorithms FA and GSO. For future works, we are aiming to enhance the performance of the 


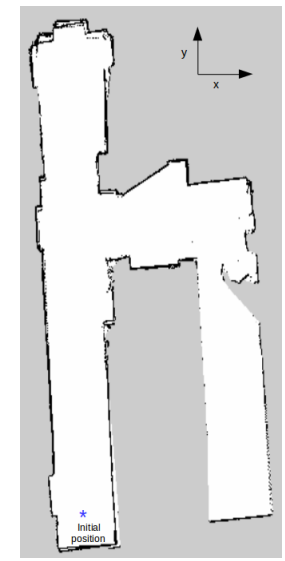

Fig. 20. Particle Swarm Optimization result in experimental environment I

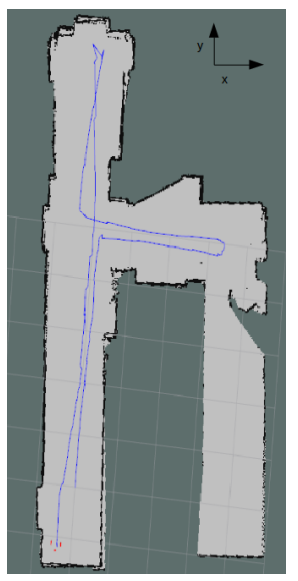

Fig. 23. Trajectory of the Particle Swarm Optimization result in experimental environment I

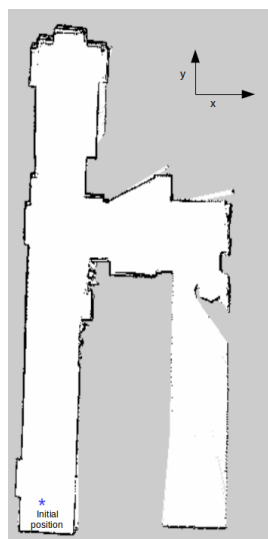

Fig. 21. Firefly Optimization Algorithm result in experimental environment I

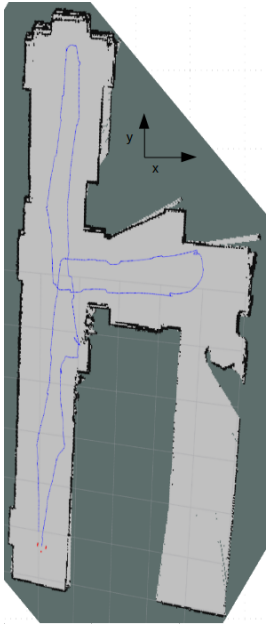

Fig. 24. Trajectory of the Firefly Optimiza- Fig. 25. Trajectory of the ion Algorithm result Glowworm Swarm Optition Algorithm result mization result in experiin experimental envi- mental environment I ronment I

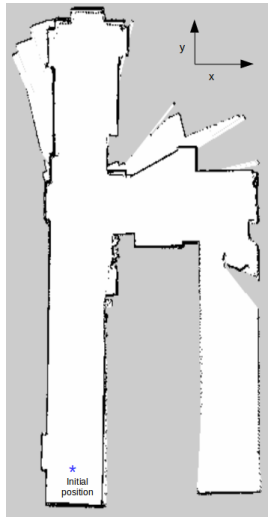

Fig. 22. Glowworm Swarm Optimization result in experimental environment I

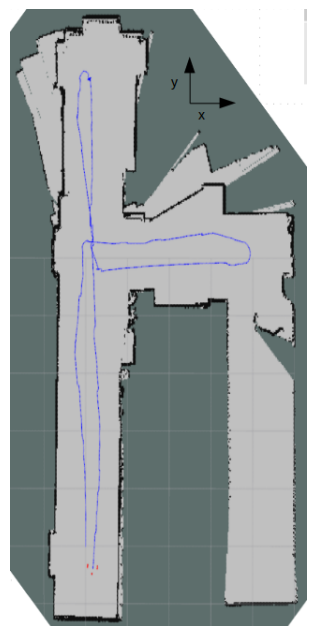

[6] Michael Lohrer, "A comparison between the firefly algorithm and particle swarm optimization ", Honors College Theses, Oakland University, pp. 11-16, Diss, 2013.

[7] Yuhui Shi, and Russell Eberhart, "A modified particle swarm optimizer," Proc.IEEE Int. Conf. on Evolutionary Computation ”, 1998.

[8] R. Eberhart, J. Kennedy, "A new optimizer using particle swarm theory ", in: Proceedings of the Sixth International Symposium on Micro Machine and Human Science, 1995.

[9] M. Quigley, K. Conley, B. Gerkey, J. Faust, T. Foote, J. Leibs, "ROS: an open-source Robot Operating System ", In ICRA workshop on open source software (Vol. 3, No. 3.2, p. 5), 2009.

[10] Kalaiselvi, T., P. Nagaraja, and Z. Abdul Basith. "A Review on Glowworm Swarm Optimization", International Journal of Information Technology (IJIT) 3.2, 2017.

[11] Kazuaki Okada, and Yasutaka Fujimoto, "Grid-based localization and mapping method without odometry information ", IEEE Industrial Electronics Society, proc. IEEE Industrial Electronics Society Annual Conference (IECON), PP.159-164, 2011.

[12] Mounia Janah, and Yasutaka Fujimoto, "Study Comparison between Firefly Algorithm and Particle Swarm Optimization for SLAM Problems", IEEJ Int. Power Electronics Conference (IPEC), 22C1-4, Niigata, 2018.5.

[13] Nakamura Yuta, and Yasutaka Fujimoto. "Validation of SLAM without odometry in outdoor environment", Advanced Motion Control (AMC), 2014 IEEE 13th International Workshop on. IEEE, 2014.

[14] Madhubhashi Senanayake, and Ilankaikone Senthooran, Jan Carlo Barca, et al. "Search and tracking algorithms for swarms of robots: A survey ", Robotics and Autonomous Systems, 2016, vol. 75, p. 422-434.

[5] Hugh Durrant-Whyte, Fellow, IEEE, and Tim Bailey "1Simultaneous Localisation and Mapping (SLAM): Part I The Essential Algorithms", 2006. 